\title{
A FILTER CHARACTERIZATION OF REGULAR BAIRE SPACES
}

\author{
R. A. $\mathrm{McCOY}$
}

ABstract. A filter characterization of regular Baire spaces is given.

Many topological properties can be defined or characterized in terms of filters. Such characterizations often prove to be useful in certain situations. We prove the following theorem which gives a filter characterization of regular Baire spaces.

THEOREM. If $X$ is a regular space, then the following are equivalent.

(i) $X$ is a Baire space.

(ii) Every point finite open filter base $\mathscr{F}$ on $X$ is locally finite at a dense set of points of $\bigcup \mathscr{F}$.

(iii) Every countable, point finite, regular open filter base $\mathscr{F}$ on $X$ is locally finite at a dense set of points of $\bigcup \mathscr{F}$.

(iv) Every countable, point finite, regular open filter base $\mathscr{F}$ on $X$ which is not locally finite at any point of $\bigcup \mathscr{F}$ has a cluster point.

A space $X$ is a Baire space provided that every nonempty open subset of $X$ is of second category in $X$. As is well known, this definition is equivalent to the statement that the intersection of a countable number of dense open subsets of $X$ is dense in $X$.

A space $X$ is lightly compact (or feebly compact) provided every locally finite collection of open sets of $X$ is finite.

An open filter base $\mathscr{F}$ on a space $X$ is a nonempty collection of nonempty open subsets of $X$ such that whenever $U$ and $V$ are members of $\mathscr{F}$, then there exists a member $W$ of $\mathscr{F}$ with $W \subset U \cap V . \mathscr{F}$ is regular if whenever $U$ is a member of $\mathscr{F}$, then there exists a member $V$ of $\mathscr{F}$ with $\bar{V} \subset U$.

LEMMA 1 ([2] AND [3]). The following are equivalent.

(i) $X$ is a Baire space.

(ii) Every point finite open cover of $X$ is locally finite at a dense set of points.

Received by the editors July 14, 1972 and, in revised form, October 12, 1972.

AMS (MOS) subject classifications (1970). Primary 54D99.

Key words and phrases. Baire spaces, open filter bases, lightly compact.

(c) American Mathematical Society 1973 
(iii) Every countable point finite open cover of $X$ is locally finite at a dense set of points.

LEMMA 2 [1]. A space $X$ is lightly compact if and only if every countable open cover of $X$ has a finite subcollection whose closures cover $X$.

LEMMA 3. Every regular lightly compact space is a Baire space.

Proof. Let $X$ be a regular lightly compact space. Let $\left\{U_{i}\right\}_{i=1}^{\infty}$ be a sequence of dense open subsets of $X$, and let $U$ be an open subset of $X$. It suffices to show that $U \cap\left(\cap_{i=1}^{\infty} U_{i}\right) \neq \varnothing$. Let $V_{1}$ be a nonempty open subset of $X$ such that $\operatorname{cl}\left(V_{1}\right) \subset U \cap U_{1}$. By induction define nonempty open subsets $V_{i}$ of $X$ such that $\operatorname{cl}\left(V_{i+1}\right) \subset V_{i} \cap U_{i+1}$ for each $i$. Suppose $\bigcap_{i=1}^{\infty} V_{i}=$ $\varnothing$. Then define $W_{i}=X \mid \operatorname{cl}\left(V_{i}\right)$, so that $\left\{W_{i}\right\}_{i=1}^{\infty}$ is a countable open cover of $X$. By Lemma 2, there exists $i_{1}<\cdots<i_{n}$ such that $\left\{\operatorname{cl}\left(W_{i_{k}}\right)\right\}_{k=1}^{n}$ covers $X$. But $\varnothing=\bigcap_{k=1}^{n} X \mid \operatorname{cl}\left(W_{i_{k}}\right) \supset \bigcap_{k=1}^{n} V_{i_{k}}=V_{i_{n}}$, which is a contradiction. Thus $U \cap\left(\bigcap_{i=1}^{\infty} U_{i}\right) \supset \bigcap_{i=1}^{\infty} V_{i} \neq \varnothing$.

We now prove the Theorem. Since an open subspace of a Baire space is a Baire space, $\bigcup \mathscr{F}$ is a Baire space for any open filter base $\mathscr{F}$ on some Baire space. Therefore it follows from Lemma 1 that (i) implies (ii). Clearly (ii) implies (iii) and (iii) implies (iv). Finally, that (iv) implies (i) will be established by the contrapositive.

If $X$ is not a Baire space, then it has a nonempty open subspace $U$ which is of first category in $X$, and hence in itself. Therefore, by Lemma 3, $U$ is not lightly compact. Thus there exists a countably infinite, locally finite collection, $\left\{W_{i}\right\}$, of nonempty open subsets of $U$. Each $W_{i}$ must be of first category in $X$. Hence, for each $i$, there exists a sequence $\left\{W_{i j}\right\}$ of nowhere dense subsets of $X$ such that $W_{i}=\bigcup_{j=1}^{\infty} W_{i j}$; also let $W_{i 0}=\varnothing$. If $X$ is regular, for each $i$, there is a sequence $\left\{V_{i j}\right\}$ of nonempty open subsets of $X$ such that $V_{i 1}=W_{i}$ and, for each $j, \operatorname{cl}\left(V_{i, j+1}\right) \subset V_{i j}$. Then for each $i$ and $j$, define

$$
U_{i j}=\left(\bigcup_{k=i}^{\infty} V_{k i}\right) \bigvee\left(\bigcup_{n=0}^{j} \bigcup_{k=0}^{n} \operatorname{cl}\left(W_{i+j-n, k}\right)\right) .
$$

Let $\mathscr{F}$ be the collection $\left\{U_{i j} \mid i \geqq 1, j \geqq 0\right\}$, which is a countable collection of nonempty open subsets of $X$. Note that $U_{i j} \subset U_{i_{1}, j_{1}} \cap U_{i_{2}, j_{2}}$, where $i=\max \left\{i_{1}, i_{2}\right\}$ and $j=\max \left\{j_{1}, j_{2}\right\}$, so that $\mathscr{F}$ is a countable open filter base on $X$.

To see that $\mathscr{F}$ is point finite, let $x \in \bigcup \mathscr{F}$. Since $\bigcup \mathscr{F}=\bigcup_{i=1}^{\infty} W_{i}, x \in$ $W_{i}$ for some $i$; let $i_{0}$ be the largest such $i$. Then there is a $j_{0}$ such that $x \in W_{i_{0}, j_{0}}$. If $i>i_{0}$, then clearly $x \notin U_{i j}$ for any $j$. On the other hand, if $i \leqq i_{0}$ and $j>j_{0}+i_{0}$, then $x \notin U_{i j}$-which can be seen by letting $n=i+j-i_{0}$ and $k=j_{0}$ in the definition of $U_{i j}$. Therefore $x$ is in only finitely many $U_{i j}$. 
To see that $\mathscr{F}$ is regular, let $U_{i j} \in \mathscr{F}$. Then

$$
\operatorname{cl}\left(U_{i+j+1, j}\right) \subset \operatorname{cl}\left(\bigcup_{k=i+j+1}^{\infty} V_{k, i+j+1}\right) .
$$

But since $\left\{W_{i}\right\}$ is locally finite, then $\left\{V_{k, i+j+1} \mid k \geqq i+j+1\right\}$ is locally finite. Therefore

$$
\operatorname{cl}\left(\bigcup_{k=i+j+1}^{\infty} V_{k, i+j+1}\right)=\bigcup_{k=i+j+1}^{\infty} \operatorname{cl}\left(V_{k, i+j+1}\right)
$$

Also each $\operatorname{cl}\left(V_{k, i+j+1}\right) \subset V_{k i}$, so that

$$
\operatorname{cl}\left(U_{i+j+1, j}\right) \subset \bigcup_{k=i+j+1}^{\infty} V_{k i} \subset U_{i j} .
$$

To see that $\mathscr{F}$ is not locally finite at any point of $\bigcup \mathscr{F}$, let $x \in \bigcup \mathscr{F}$, and let $W$ be a neighborhood of $x$. For every $i$ and $n, W$ is not contained in $\bigcup_{k=0}^{n} \operatorname{cl}\left(W_{i k}\right)$ because each $W_{i k}$ is nowhere dense in $X$. Then since

$$
U_{1 j}=\left(\bigcup_{k=1}^{\infty} W_{k}\right) \bigvee\left(\bigcup_{n=0}^{j} \bigcup_{k=0}^{n} \operatorname{cl}\left(W_{1+j-n, k}\right)\right)
$$

$W$ must intersect every $U_{1 j}$.

To see that $\mathscr{F}$ cannot have a cluster point, let $x \in X$. Since $\left\{W_{i}\right\}$ is locally finite, there exist an integer $n$ and neighborhood $W$ of $x$ which intersects no $W_{i}$ for $i \geqq n$. This means that $x \notin \bigcup_{k=n}^{\infty} \operatorname{cl}\left(W_{k}\right)$. But $U_{n 1} \subset$ $\bigcup_{k=n}^{\infty} W_{k}$, so that $\operatorname{cl}\left(\bar{U}_{n 1}\right) \subset \operatorname{cl}\left(\bigcup_{k=n}^{\infty} W_{k}\right)$. Again because of the local finiteness of $\left\{W_{i}\right\}, \operatorname{cl}\left(\bigcup_{k=n}^{\infty} W_{k}\right)=\bigcup_{k=n}^{\infty} \operatorname{cl}\left(W_{k}\right)$. Therefore $x \notin \operatorname{cl}\left(U_{n 1}\right)$, so that $x$ cannot be a cluster point of $\mathscr{F}$.

Note that the hypothesis that $X$ be a regular space in the Theorem and in Lemma 3 can be weakened to $X$ being a quasi-regular space; that is, for every nonempty open $U$ in $X$, there is a nonempty open $V$ in $X$ with $\bar{V} \subset U$.

\section{REFERENCES}

1. R. W. Bagley, E.H. Connell, and J. D. McKnight, Jr., On properties characterizing pseudo-compact spaces, Proc. Amer. Math. Soc. 9 (1958), 500-506. MR 20 \#3523.

2. P. Fletcher and W. F. Lindgren, A note on spaces of second category, (to appear).

3. R. A. McCoy, A Baire space extension, Proc. Amer. Math. Soc. 33 (1972), 199-202.

Department of Mathematics, Virginia Polytechnic Institute and State UniVERSITY, BLACKSBURG, VIRGINIA 24061 